Júlia de Souza Baptista

(ㄱ) https://orcid.org/0000-0002-4730-5800

Tatiana Henriques Leite²

Ohttps://orcid.org/0000-0002-2861-4480

Vanessa Karam de Lima Ferreira

(-) https://orcid.org/0000-0001-5534-1484

Luciana de Souza Pereira de

Magalhães

(1) https://orcid.org/0000-0002-6937-4989

Marcelo Santos Cruz

(D) https://orcid.org/0000-0003-0057-2095

Ana Carolina Robbe Mathias Ohttps://orcid.org/0000-0003-0859-3704

\section{Relationship between levels of mindfulness and substance use pattern}

\author{
Relação entre níveis de mindfulness e padrão do uso de substâncias
}

DOI: 10.1590/0047-2085000000337

\begin{abstract}
Objective: Evaluate the association between levels of mindfulness and sociodemographic characteristics and pattern of drug use of individuals seeking treatment in a University Service Specialized in Substance Use Disorders. Methods: This is a cross-sectional study with 164 individuals over 18 years of age seeking treatment for the use of psychoactive substances in the June 2018-December 2019 period, using a questionnaire for sociodemographic data, the Mindful Attention Awareness Scale (MAAS) self-reporting instrument, and the Alcohol, Smoking, and Substance Involvement Screening Test. Results: An association was found between low levels of mindfulness mainly with the individual risk of being a medium/high-risk user of sedative-hypnotic drugs $(p=0.020)$. A borderline association was also found between MAAS and the risk of the individual being a medium/high risk of alcohol ( $p=0.053$ ) and with a more severe pattern of substance use $(p=0.065)$. Conclusion: Individuals seeking treatment for substance use presented impairments in the attentional aspect of mindfulness and levels of mindfulness seem to protect against behaviors related to substance use, especially against the use of high/ moderate risk of sedative-hypnotics.
\end{abstract}

\section{KEYWORDS}

Mindfulness, substance use disorders, mindfulness levels, substance use severity, substance abuse.

\section{RESUMO}

Objetivo: Avaliar a associação entre níveis de mindfulness e características sociodemográficas e padrão do uso de drogas de indivíduos que buscam tratamento em Serviço Universitário Especializado em Transtorno por Uso de Substâncias. Métodos: Estudo de corte transversal de 164 indivíduos acima de 18 anos que buscavam tratamento para uso de substâncias psicoativas no período de junho de 2018 a dezembro de 2019, utilizando questionário para dados sociodemográficos, o instrumento de autorrelato Mindful Attention Awareness Scale (MAAS) e o Alcohol, Smoking and Substance Involvement Screening Test. Resultados: Foi encontrada associação entre baixos níveis de mindfulness principalmente com o risco de o indivíduo ser usuário de médio/alto risco de sedativos-hipnóticos ( $p=0,020)$. Também foi encontrada associação limítrofe entre MAAS com risco de o indivíduo ser usuário de médio/alto risco de álcool ( $p=0,053)$ e com padrão mais grave de uso de substâncias $(p=0,065)$. Conclusão: Indivíduos que buscavam tratamento para uso de substâncias apresentaram prejuízos no aspecto atencional de mindfulness, e níveis de mindfulness parecem proteger contra comportamentos relacionados ao uso de substâncias, principalmente contra o uso de alto/moderado risco de sedativos-hipnóticos.

PALAVRAS-CHAVE

Mindfulness, transtorno por uso de substâncias, níveis de mindfulness, gravidade do uso de substâncias, abuso de substância. 


\section{INTRODUCTION}

Substance use disorder (SUD) is a prevalent public health problem that leads to severe social and personal consequences'. In 2018, about 269 million people worldwide used psychoactive substances at least once in the previous year, which corresponds to $5.4 \%$ of the world population aged 15-64 years. Among them, 35.6 million people suffer from SUDs, which means that their psychoactive substances pattern is harmful, they may experience psychoactive substances dependence, or require treatment ${ }^{2}$.

Regarding the Brazilian population, a recent epidemiological study estimated that $1.5 \%$ of individuals had alcohol dependence criteria and 2.2\% for alcohol and a substance other than tobacco. Regarding the use of illicit substances, about $7.7 \%$ of Brazilians reported having used marijuana at least once in their lives, and the second-most illicit substance consumed was powder cocaine $3.1 \%$ reported consuming the drug) ${ }^{3}$.

In Brazil, the most consumed medication in a nonprescribed way or differently from that prescribed were the Benzodiazepines (BZD), with a prevalence of 3.9\% at least once in the lifetime ${ }^{3}$. Also, more than $90 \%$ of BZD users do so through a medical prescription ${ }^{4}$, despite consensus on the risks involved with its prolonged use. BZDs are used as hypnotics and sedatives ${ }^{5}$, and the ability to generate tolerance and dependence can be caused by inadequate and continued doctor's prescription, patient self-increased dose $^{6}$, and cognitive and psychological factors such as the anticipation of adverse effects from a bad night's sleep or a malfunction the next day, leading to higher levels of anxiety ${ }^{7}$. Also noteworthy is the fear of having withdrawal symptoms with the suspended or even reduced use of these substances ${ }^{5}$.

SUD patients show impaired consciousness and clarity of their emotions, difficulty controlling impulses while experiencing negative emotions ${ }^{8}$, and often use dysfunctional emotional regulation strategies. Attentional control, behavioral monitoring, interoceptive awareness and self-regulation may modulate the impaired insight, automatic habits and stress reactivity that promote addictive behavior?. SUD treatment is still a challenge in clinical practice and, thus, further studies should be carried out to better understand the characteristics of these individuals and the factors involved in a more severe substance use pattern to establish effective therapeutic measures.

The number of studies on interventions that can reduce substance use and prevent relapses has grown in the last decades. In particular, mindfulness-based therapies are indicated in the literature as potential intervention ${ }^{10}$ among them, Mindfulness-Based Relapse Prevention ${ }^{11}$, MindfulnessOriented Recovery Enhancement' ${ }^{2}$, Modified Mindfulness Training for Smoking Cessation ${ }^{13}$ and Vipassana Meditation ${ }^{14}$.
Mindfulness can be characterized as a natural dispositional trait among people and involves perceiving stimuli (thoughts, sensations, and feelings) simply "as they are ${ }^{\prime \prime 14}$, and is a capacity inherent to human beings that varies in the general population, even in the absence of training ${ }^{15}$. Besides a trait, with dispositional characteristic, Mindfulness meaning also may be a state, with a situational and transitory nature and a practice, consisting in practices based on mindfulness ${ }^{16}$. There are reasons to expect that level of mindfulness, as a practice, can be associated with fewer problems related to substance use. First, some studies suggest that mindfulness can lead to fewer relapse episodes and fewer negative consequences of substance use $\mathrm{e}^{17}$. Mindfulness is linked to lower levels of fear and avoidance of internal experiences such as emotions and cognitions. It can reduce substance use motivated by the desire to avoid unpleasant thoughts and feelings ${ }^{18,19}$. One addictive process that is likely offset by trait mindfulness is the addiction attentional bias, in other words, preferential attention to substance-relevant cues that have been conferred incentive salience through mesocorticolimbic sensitization ${ }^{20}$. The first meta-analysis to quantify the relationship between trait mindfulness and substance use behaviors and at determining how this relationship varies in context of substance type, found out that the relationships of trait mindfulness were more strongly related with alcohol and tobacco use compared to marijuana use behaviors ${ }^{21}$. These findings might be due to some characteristics of these substances, like cue reactivity and cravings. In this study ${ }^{21}$, prescription medication, sedative, or stimulant use behaviors were not included in the analysis.

Concerning the use of sedatives-hypnotics, level of mindfulness seems to be inversely related to the concern about the availability of medications and the noncompliance with the proposed therapeutic regimen with their use $^{22}$. More conscious individuals could address withdrawal-related distress than individuals with low levels of mindfulness ${ }^{23}$, since individuals with high levels of mindfulness tend to have a better ability to see aversive experiences as transient ${ }^{24,25}$ and making more skillful behavioral choices, reducing the need to relieve physical, cognitive, or emotional discomfort with substance use ${ }^{11}$. In contrast to mindfulness, which is associated with cognitive and behavioral flexibility, behaviors related to the use of psychoactive substances can be characterized by a state of mindlessness ${ }^{26}$, that is, in which people exhibit habitual or stereotyped autopilot responses ${ }^{23}$. Garland investigated the association between trait mindfulness and alcohol cue-reactivity ${ }^{27}$, and found that among alcohol-dependent adults, higher trait mindfulness was linked with an increased capacity to reorient attention away from alcohol cues as well as greater recovery of heart hate variability following stressprimed alcohol cue exposure ${ }^{28}$. 
There is still a lack of studies on mindfulness deficits among individuals seeking treatment for substance abuse ${ }^{29}$ other than intervention studies. One recent study found that individuals seeking treatment for SUD had lower score of Mindful Attention Awareness Scale (MAAS) than the national average, $4.22(\mathrm{SD}=.63)$ and multiple-substance users, 3.6 $(S D=1.2)$ had even lower levels of mindfulness than singlesubstance users, $4(\mathrm{SD}=1)^{30}$.

The literature also shows that low levels of mindfulness have been related to greater severity of the substance use pattern $^{31}$. This relationship between mindfulness and substance use seems to be more robust between hospitalized individuals and those with problematic substance use ${ }^{31}$. Also, some studies suggest that there is no difference between the levels of mindfulness of men and women seeking treatment for SUD 29,32 .

Given the above, understanding the relationship between levels of mindfulness and substance use can better understand the degree of protection provided by mindfulness against substance use and the clinical usefulness of mindfulness-based training or interventions in reducing substance use. Thus, this study aims to assess whether individuals seeking treatment for SUD with low levels of mindfulness have a more severe pattern of drug use and verify whether this relationship varies according to sociodemographic characteristics and type of substance used. We therefore used the MAAS to investigate this relationship, which is a unidimensional short scale and, according to its original version ${ }^{33}$, measures levels of mindfulness. More specifically, this instrument assesses individual differences in the frequency of mindful states over time, focusing on the core attentional aspect of mindfulness ${ }^{33}$.

\section{METHODS}

\section{Ethical aspects}

The Research Ethics Committee of the Psychiatry Institute of the Federal University of Rio de Janeiro approved the study protocol under Opinion CAAE 89088218.0.0000.5263.

\section{Procedures}

Data collected between June 14, 2018, and December 12, 2019, from 164 patients seeking treatment in the Drug Abuse Research and Assistance Program (PROJAD) for substance use-related behaviors were analyzed. The inclusion criteria for the research participants were: (1) patients with problems related to the use of psychoactive substances; (2) patients entering specialized treatment for substance use-related behaviors; (3) age above 18 years, and (4) who agreed to participate in the study by signing the Informed Consent Form (ICF). The following were excluded: (1) patients who are unable, for some reason, to answer the scales/questionnaires requested by the research and (2) individuals under 18 years of age.

\section{Instruments}

All patients who arrived at the PROJAD reception and agreed to participate in the research were submitted to the following instruments:

1. ICF;

2. Registration form for the sociodemographic survey. Sociodemographic data investigated: sex, age, religion, marital status, educational level, occupation, income, travel expenses for the treatment site, legal matters, time of drug use, family members with drug problems, treatment for other disorders and perception related to another compulsion (shopping, sex, gaming/gambling, and internet);

3. Alcohol, Smoking, and Substance Involvement Screening Test (ASSIST). A structured questionnaire containing eight questions about the use of nine psychoactive substances classes (tobacco, alcohol, marijuana, cocaine, sedatives, stimulants, inhalants, hallucinogens, and opiates). It aims to screen problematic psychoactive substances use cases. Information on substance use and the problems related to that use throughout life and in the last three months is collected. It also allows to classify the severity of the risk of having problems related to substance use (low, moderate, or suggestive of addiction) $)^{34}$. The internal consistency of the instrument is good, assessed by Cronbach's alpha coefficient, with coefficients: .80 for alcohol, .80 for tobacco, .79 for marijuana and .81 for cocaine ${ }^{34}$;

4. Mindful Attention Awareness Scale (MAAS) - It is a self-report questionnaire with good reliability ratings: total scale (Cronbach alpha .83) and test-retest (Pearson .80) (35 $^{35}$ that measures mindfulness levels focusing on the presence or absence of attention and awareness of what is happening in the present moment. It consists of 15 items on a Likert scale ranging from (1) almost always to (6) rarely, with the highest scores indicating higher levels of mindfulness. Participants are asked to point out how often they experience each situation described and not how they think the experience should be. The scale was translated and validate in Portuguese ${ }^{35}$. The items are distributed among general, cognitive, emotional, physical, and interpersonal domains ${ }^{33}$.

\section{Statistical analyses}

First, a univariate analysis was conducted to describe the study's population considering sociodemographic, 
psychiatric history, and drug use. A bivariate analysis was performed to show differences between levels of mindfulness and the mentioned characteristics according to variables presentation (T-test, ANOVA, or Pearson correlation). P-valor minor or equal to 0,05 was used to indicate a difference between groups.

The association between levels of mindfulness (exposure) and drug use (outcome) was investigated using a linear regression model to continuum outcome (drug use-total). A logistic regression analysis was employed for dichotomy outcomes (types of drugs). The analysis considered a full model (levels of mindfulness, education, age, gender, personal income, previous psychiatric treatment, previous hospitalization, number of drugs used, and compulsive behavior). The variable with the highest $p$-value was excluded, and the model was re-specified. The nested models were compared using R2 (higher is better). All the analyses were performed using Stata Statistical Software 13.0. In this analysis, p-valor minor or equal to 0,05 was used to indicate statistical significance; and values higher than 0,05 and minor than 0,1 as a borderline.

\section{RESULTS}

Table 1 presents the sociodemographic characteristics of the 164 participants. We did not find an association between higher MAAS scores and sociodemographic characteristics. MAAS scores were associated with the highest total ASSIST score $(p=0.019)$, with previous hospitalization history ( $p=$ $0.034)$ and use of sedative-hypnotic drugs ( $p=0.065)$.

Table 2 shows a significant association between MAAS and the individual's risk of being a medium/high-risk hypnotic sedative user $(p=0.02)$. We observed that MAAS is associated with lower risk of individuals being medium/ high-risk alcohol users $(p=0.053)$.

\section{DISCUSSION}

Mindfulness deficits have been common in substanceusing populations ${ }^{30}$. Our study found similar findings to the literature. The main results indicate that higher levels of mindfulness are associated with a lower likelihood of individuals to be medium/high-risk users of sedativeshypnotics. Consistent with previous studies ${ }^{30,31}$, levels of mindfulness were inversely associated with the pattern of substance use in our sample. The discrete inverse association of levels of mindfulness with the risk of medium/high risk of alcohol use may be explained by some of its characteristics related to the process of dependence on this substance, such as fissure and reactivity towards triggers ${ }^{36}$. Persons who present higher levels of trait mindfulness are more capable to disengage attentional resources from processing alcoholrelated cognitions ${ }^{27}$.

The first study to assess the relationship between dispositional mindfulness and sedative-hypnotic drug addiction aspects among chronic female users of these substances indicated possible protection of dispositional mindfulness for sedative-hypnotic drug addiction ${ }^{22}$. In that same study, they observed that dispositional mindfulness was inversely associated with some aspects of benzodiazepine (BZD) dependence. These aspects are (a) an anticipatory cognitive component related to the possible unavailability of medication; (b) compulsive component of medication use beyond the prescribed dose; (c) anxiety about suffering from symptoms if the indicated dose does not lead to the expected results; (d) the perception of the damage caused by the withdrawal symptoms in the case of reduction or suspension of the use of sedatives-hypnotics. Another important finding of the same study was that there was no relationship between dispositional mindfulness and perception of sedatives-hypnotics' problematic use. This relationship can perhaps be explained by the fact that most medications are provided under medical supervision ${ }^{22}$.

The literature suggests an increasing tendency to incorporate sedative-hypnotic drugs as medicines routinely prescribed to relieve depressive, anxiolytic, and hypnotic symptoms ${ }^{37}$. It also indicates that women are part of the group that most consumes such substances to increase use with age. This trend may be related to increasing insomnia cases with age $^{5}$. Anticipatory anxiety related to a bad night's sleep, with consequences the next day, such as low work productivity, can culminate in a medication use beyond the prescribed dose. On the other hand, individuals with high dispositional mindfulness traits are expected to be aware of these concerns in a non-reactive way, understanding that they are transient.

BZDs are widely known for their potential to generate tolerance, dependence, withdrawal syndrome with prolonged use, and cause cognitive deficits (for example, in memory, attention, and processing speed $)^{6,38}$.

One might think that this negative relationship between levels of mindfulness and SUD severity is reciprocal so that low levels of mindfulness would be associated with less protection against SUD ${ }^{14}$ and SUD would decrease mindfulness levels. In particular, this could occur because mindfulness involves the use of higher-order cognitive processes $^{39}$. However, these are hypotheses that cannot be analyzed in cross-sectional studies, given the limitations of this type of study to determine causalities.

The results of this study and the literature suggest that mindfulness-based interventions may be useful for individuals who use substances to relieve emotional, physical, or mental discomfort ${ }^{40}$. The ability to perceive internal and external experiences with the awareness that they are transient allows individuals to adopt a more 
conscious attitude and not react from them. Likewise, being aware that ruminations are mental events and not absolute truths seems to allow the individual to distance himself from the ruminations, avoiding engagement.

Table 1. Descriptive and bivariate analyses of sociodemographic, psychiatric history, drug use and levels of mindfulness

\begin{tabular}{|c|c|c|c|c|}
\hline & $\mathbf{n}=$ & $\begin{array}{c}\text { Total } \\
n=164 \\
\% \text { or mean (Cl } 95 \%)\end{array}$ & Mindfulness (Score) & p-value \\
\hline $\mathrm{Age}^{*}$ & & & & 0.204 \\
\hline$\leq 30$ years & 35 & $21.7(10.4-40.1)$ & 3.1 & \\
\hline$\geq 31$ years & 126 & $78.3(70.3-85.3)$ & 3.3 & \\
\hline Gender* & & & & 0.548 \\
\hline Male & 116 & $72.1 \%(63.3-80.0)$ & 3.3 & \\
\hline Female & 46 & $27.9 \%(15.9-43.4)$ & 3.2 & \\
\hline Civil status* & & & & 0.370 \\
\hline Marriage or living together & 52 & $32.5(20.3-47.1)$ & 3.2 & \\
\hline Do not cohabits with companion & 108 & $67.5(57.9-76.2)$ & 3.3 & \\
\hline Personal income ${ }^{{ }_{2}}$ & & & & 0.321 \\
\hline Until $\mathrm{R} \$ 954$ & 46 & $28.2(15.9-43.4)$ & 3.1 & \\
\hline Above $\mathrm{R} \$ 955$ & 118 & $71.8(63.0-80.0)$ & 3.3 & \\
\hline Educational level** & & & & 0.646 \\
\hline Elementary school incomplete & 21 & $13.1(3.0-36.3)$ & 3.1 & \\
\hline Elementary school complete & 34 & $21.2(8.7-37.8)$ & 3.3 & \\
\hline High school complete & 81 & $50.6(39.2-61.9)$ & 3.2 & \\
\hline Higher education & 24 & $15.1(4.7-37.3)$ & 3.4 & \\
\hline Previous psychiatric treatment* & & & & 0.207 \\
\hline Yes & 51 & $32.0(19.1-45.8)$ & 3.3 & \\
\hline No & 108 & $68.0(57.9-76.2)$ & 3.1 & \\
\hline Previous hospitalization* & & & 3.1 & 0.034 \\
\hline Yes & 73 & $46.5(34.8-58.6)$ & 3.4 & \\
\hline No & 84 & $53.5(42.3-64.5)$ & & \\
\hline Presence of compulsive behaviour* & & & & 0.150 \\
\hline Yes & 134 & $83.2(76.2-89.4)$ & 3.2 & \\
\hline No & 27 & $16.7(6.3-38.0)$ & 3.5 & \\
\hline Tobacco use* & & & & 0.495 \\
\hline Low risk & 49 & $30.8(18.2-45.4)$ & 3.3 & \\
\hline Medium/high risk & 110 & $69.2(59.6-77.5)$ & 3.2 & \\
\hline Alcohol use* & & & 3.4 & 0.159 \\
\hline Low risk & 58 & $36.5(23.9-49.8)$ & 3.2 & \\
\hline Medium/high risk & 101 & $63.5(53.1-72-7)$ & & \\
\hline Cannabis use ${ }^{*}$ & & & & 0.350 \\
\hline Low risk & 88 & $55.3(44.6-66.2)$ & 3.3 & \\
\hline Medium/high risk & 71 & $44.7(33.2-57.3)$ & 3.2 & \\
\hline Cocaine use ${ }^{\star}$ & & & & 0.398 \\
\hline Low risk & 52 & $32.3(20.3-47.1)$ & 3.3 & \\
\hline Medium/high risk & 109 & $67.7(58.2-76.5)$ & 3.2 & \\
\hline Sedative use* & & & & 0.065 \\
\hline Low risk & 108 & $67.0(57.0-75.4)$ & 3.3 & \\
\hline Medium/high risk & 53 & $33.0(20.0-46.3)$ & 3.1 & \\
\hline Number of drugs used ** & & & & 0.442 \\
\hline $1-3$ & 24 & $15.8(4.7-37.3)$ & 3.4 & \\
\hline $4-6$ & 79 & $52.3(40.3-63.2)$ & 3.3 & \\
\hline $7-9$ & 48 & $31.7(18.6-46.2)$ & 3.2 & \\
\hline Score drug use (ASSIST - TOTAL) (mean) ${ }^{\star \star *}$ & 153 & $73.1(33.0-113.3)$ & -0.189 & 0.019 \\
\hline
\end{tabular}

Notes: ${ }^{*}$ T-test. ${ }^{* \star}$ ANOVA. ${ }^{* \star}$ Pearson correlation. ${ }^{a}$ indicates minimum wage. 
Table 2. Association between levels of mindfulness, types of drugs and total drug use among adults: logistic regression model

\begin{tabular}{|c|c|c|c|c|c|c|c|c|}
\hline \multirow[b]{2}{*}{ Mindfulness (MAAS) } & \multicolumn{4}{|c|}{ Crude } & \multicolumn{4}{|c|}{ Adjusted } \\
\hline & $\begin{array}{c}\text { Regression } \\
\text { Coefficient/OR }\end{array}$ & (95\% Cl) & Std. error & p-value & $\begin{array}{c}\text { Regression } \\
\text { Coefficient/OR }\end{array}$ & (95\% Cl) & Std. error & p-value \\
\hline Drug use (ASSIST - total)a & -8.77 & -15.7 to -1.7 & 3.5 & 0.014 & -6.32 & $-13.0-0.39$ & 3.4 & 0.065 \\
\hline Tobacco $^{b}$ & 0.87 & $0.60-1.27$ & 0.16 & 0.496 & 1.15 & $0.65-2.04$ & 0.33 & 0.617 \\
\hline Alcohol $^{\mathrm{c}}$ & 0.76 & $0.53-1.10$ & 0.14 & 0.159 & 0.66 & $0.44-1.00$ & 0.13 & 0.053 \\
\hline Cannabis $^{d}$ & 0.84 & $0.59-1.19$ & 0.15 & 0.348 & 1.09 & $0.6-2.0$ & 0.33 & 0.761 \\
\hline Cocaine $^{e}$ & 0.85 & $0.58-1.23$ & 2.35 & 0.396 & 0.94 & $0.54-1.65$ & 0.26 & 0.855 \\
\hline Sedatives ${ }^{f}$ & 0.70 & $0.48-1.02$ & 0.13 & 0.067 & 0.48 & $0.26-0.89$ & 0.15 & 0.020 \\
\hline
\end{tabular}

${ }^{a}$ Analyses adjusted by age and number of drugs used.

${ }^{\mathrm{b}}$ Analyses adjusted by education, age, personal income, previous psychiatric treatment, previous hospitalization and number of drugs used.

${ }^{c}$ Analyses adjusted by education, age, previous psychiatric treatment, previous hospitalization and presence of compulsive behaviour.

${ }^{d}$ Analyses adjusted by age, personal income, previous hospitalization, number of drugs used and presence of compulsive behaviour.

${ }^{e}$ Analyses adjusted by education, age, gender, personal income, previous hospitalization, number of drugs used and presence of compulsive behaviour.

${ }^{\dagger}$ Analyses adjusted by age, gender, number of drugs used, previous psychiatric treatment and previous hospitalization.

*Reference category: low risk user

Since dispositional mindfulness is a human skill that can be modified with mindfulness-based therapies and meditative practices, interventions that incorporate mindfulness prove to be potential treatments for SUDs. They allow the individual to become aware of the unpleasant sensations and experiences in the present moment, recognize them as transient, and not react on autopilot mode ${ }^{40,41}$. Therefore, the use of mindfulness exercises aims to help individuals interrupt

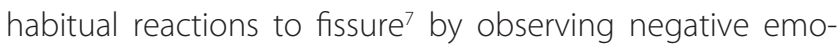
tions and impulses without reacting to them ${ }^{40}$, since substance dependent individuals exhibit automatic attentional responses to drug-related cues $^{42}$. These individuals may benefit from mindfulness exercises that focus on particular mindfulness-related impairments ${ }^{30}$, for example, improving attention and awareness to the present moment, in the case of the population of the present study.

Mindfulness exercises, such as meditation, also proved useful in the treatment associated with cognitive-behavioral therapy in the discontinuation of sedatives-hypnotics ${ }^{7}$. High dispositional mindfulness levels were associated with a lower perception of withdrawal symptoms when trying to reduce or stop the use of sedatives-hypnotics ${ }^{43}$, which suggests that dispositional mindfulness does not alter the experience itself, but the individual's relationship with it.

\section{Study limitations}

This study has some limitations. First, the cross-sectional design we used does not allow inferring causality from its findings. Another drawback is the small sample size which may have influenced the lack of significance of other substances. Measuring mindfulness levels is very challenging, since experience and knowledge with meditative practices influence the interpretation of the items in the mindfulness questionnaires, and it is not only a limitation of this study. Levels of mindfulness was measured through self-report, and its responses may have been affected if individuals had chosen alternatives that they believe are more appropriate or correct instead of those that would apply more in their case.

\section{CONCLUSION}

Mindfulness-related attentional impairments were found in individuals seeking treatment for SUDs. The identification of mindful related attentional impairments by MAAS may contribute for the adjustment of treatment schedules for patients with SUD. The relevance of the present findings may also contribute for the development of specific treatment strategies. We also found that levels of mindfulness can protect against behaviors related to substance use, especially the use of sedatives-hypnotics, alcohol, and against the use of drugs with a more severe pattern.

\section{INDIVIDUAL CONTRIBUTIONS}

Júlia de Souza Baptista - Conception, interpretation of results, writing the article, and approval of the version to be published.

Tatiana Henriques Leite - Analysis, interpretation of results, revision of the text, and approval of the version to be published.

Vanessa Karam de Lima Ferreira, Luciana de Souza Pereira de Magalhães, Ana Carolina Robbe Mathias - Project, design, revision of the text, and approval of the version to be published.

Marcelo Santos Cruz - Conception, interpretation of results, revision of the text, and approval of the version to be published. 


\section{CONFLICT OF INTERESTS}

The authors report no conflicts of interest.

\section{ACKNOWLEDGMENTS}

We are very grateful to PROJAD's trainees for the application of the questionnaires and Jean Pierre Barakat for translating this paper.

\section{DATA AVAILABILITY STATEMENT}

The data that support the findings of this study are available from the corresponding author upon reasonable request.

\section{FUNDING DETAILS}

The authors funded the study.

\section{REFERENCES}

1. Grant S, Colaiaco B, Motala A, Shanman R, Booth M, Sorbero M, et al. Mindfulness-based Relapse Prevention for Substance Use Disorders: A Systematic Review and Meta-analysis. J Addict Med. 2017;11(5):386-96.

2. United Nations Publications. Booklet 2: Drug Use and Health Consequences. World Drug Report; 2020.

3. Bastos FIPM, Vasconcellos MTL, De Boni RB, Reis NB, Coutinho CFS. III Levantamento Nacional Sobre o Uso de Drogas pela População Brasileira. Rio de Janeiro: Fiocruz/ICICT; 2017.

4. Kapczinski F, Amaral OB, Madruga M, Quevedo J, Busnello JV, de Lima MS. Use and misuse of benzodiazepines in Brazil: a review. Subst Use Misuse. 2001;36(8):1053-69.

5. Fiorelli K, Assini FL. A prescrição de benzodiazepínicos no Brasil: uma análise da literatura. ABCS Health Sci [Internet]. 2017 Apr 26;42(1):40-4. Available from: https://www.portalnepas. org.br/abcshs/article/view/948

6. Nordon DG, Hübner KC. Prescrição de benzodiazepínicos por clínicos gerais. Diagn Tratamento. 2009;14(2):66-9

7. Lunde LH, Skjøtskift S. Combining mindfulness meditation with cognitive behavior therapy and medication taper for hypnotic-dependent insomnia in older adults: A case study. Clin Case Stud. 2015;14(4):307-20.

8. Fox HC, Axelrod SR, Paliwal P, Sleeper J, Sinha R. Difficulties in emotion regulation and impulse control during cocaine abstinence. Drug Alcohol Depend. 2007;89(2-3):298-301.

9. Goldstein RZ, Craig AD, Bechara A, Garavan H, Childress AR, Paulus MP, et al. The Neurocircuitry of Impaired Insight in Drug Addiction. Trends Cogn Sci. 2009;13(9):372-80.

10. Chiesa A, Serretti A. Are Mindfulness-Based Interventions Effective for Substance Use Disorders? A Systematic Review of the Evidence. Subst Use Misuse. 2014;49(5):492-512.

11. Bowen S, Chawla N, Collins SE, Witkiewitz K, Hsu S, Grow J, et al. Mindfulness-based relapse prevention for substance use disorders: A pilot efficacy trial. Subst Abus. 2009;30(4):295-305.

12. Garland EL, Gaylord SA, Boettiger CA, Howard MO. Mindfulness Training Modifies Cognitive, Affective, and Physiological Mechanisms Implicated in Alcohol Dependence: Results of a Randomized Controlled Pilot Trial. J Psychoactive Drugs. 2010;42(2):177-92.

13. Davis JM, Goldberg SB, Anderson MC, Manley AR, Smith SS, Baker TB. Randomized Trial on Mindfulness Training for Smokers Targeted to a Disadvantaged Population. Subst Use Misuse. 2014:49(5):571-85.
14. Marlatt GA, Witkiewitz K, Dillworth TM, Bowen SW, Parks GA, et al. Vipassana meditation as a treatment for alcohol and drug use disorders. In: Steven $\mathrm{CH}$, Victoria MF, Marsha ML, editors. Mindfulness and acceptance: Expanding the cognitive-behavioral tradition. El Paso, Texas: University of Texas/Guildford Press; 2004

15. Brown KW, Ryan RM. The benefits of being present: Mindfulness and its role in psychological well-being. J Pers Soc Psychol. 2003;84(4):822-48.

16. Baer RA. Measuring mindfulness. Contemp Buddhism [Internet]. 2011 May 1;12(1):241-61. Available from: http://www.tandfonline.com/doi/abs/10.1080/14639947.2011.564842

17. Lucena-Santos P, Pinto-Gouveia J, da Silva Oliveira M. Terapias comportamentais de terceira geração [Internet]. 2015. Available from: https://www.sinopsyseditora.com.br/ upload/produtos_pdf/370.pdf

18. Zgierska A, Rabago D, Chawla N, Kushner K, Koehler R, Marlatt A. Mindfulness meditation for substance use disorders: A systematic review. Subst Abus. 2009;30(4):266-94.

19. Baer RA, Smith GT, Hopkins J, Krietemeyer J, Toney L. Using self-report assessment methods to explore facets of mindfulness. Assessment. 2006;13(1):27-45.

20. de Souza I, Kozasa E, Rabello L, Mattozo B, Bowen S, Richter K, et al. Dispositiona mindfulness, affect and tobacco dependence among treatment naive cigarette smokers in Brazil. Tob Induc Dis. 2019;17:28.

21. Robinson TE, Berridge KC. Review. The incentive sensitization theory of addiction: some current issues. Philos Trans R Soc Lond B Biol Sci. 2008:363(1507):3137-46.

22. Karyadi KA, VanderVeen JD, Cyders MA. A meta-analysis of the relationship between trait mindfulness and substance use behaviors. Drug Alcohol Depend. 2014;143(1):1-10.

23. Barros VV, Opaleye ES, Demarzo M, Bowen S, Curado DF, Hachul H, et al. Dispositional mindfulness, anticipation and abstinence symptoms related to hypnotic dependence among insomniac women who seek treatment: A cross-sectional study. PLoS One. 2018;13(3):e0194035.

24. Garland EL, Hanley AW, Thomas EA, Knoll P, Ferraro J. Low dispositional mindfulness predicts self-medication of negative emotion with prescription opioids. J Addict Med. 2015;9(1):61-7.

25. Brewer JA, Bowen S, Smith JT, Marlatt GA, Potenza MN. Mindfulness-based treatments for co-occurring depression and substance use disorders: what can we learn from the brain? Addiction. 2010;105(10):1698-706.

26. Kabat-Zinn J. Mindfulness-Based Interventions in Context: Past, Present, and Future. Clin Psychol Sci Pract [Internet]. 2003 May 1;10(2):144-56. Available from: http://doi.wiley. com/10.1093/clipsy/bpg016

27. Langer EJ. Matters of mind: Mindfulness/mindlessness in perspective. Conscious Cogn [Internet]. 1992 Sep;1(3):289-305. Available from: https://linkinghub.elsevier.com/ retrieve/pii/105381009290066J

28. Garland EL. Trait mindfulness predicts attentional and autonomic regulation of alcohol cue-reactivity. J Psychophysiol. 2011;25(4):180-9.

29. Garland EL, Roberts-Lewis A, Kelley K, Tronnier C, Hanley A. Cognitive and Affective Mechanisms Linking Trait Mindfulness to Craving Among Individuals in Addiction Recovery. Subst Use Misuse. 2014:49(5):525-35.

30. Shorey RC, Brasfield H, Anderson S, Stuart GL. Mindfulness deficits in a sample of substance abuse treatment seeking adults: a descriptive investigation. J Subst Use. 2014;19(1-2):194-8.

31. Dakwar E, Mariani JP, Levin FR. Mindfulness impairments in individuals seeking treatment for substance use disorders. Am J Drug Alcohol Abuse. 2011;37(3):165-9.

32. Bowen S, Enkema MC. Relationship between dispositional mindfulness and substance use: Findings from a clinical sample. Addict Behav. 2014;39(3):532-7.

33. Brooks M, Kay-Lambkin F, Bowman J, Childs S. Self-compassion amongst clients with problematic alcohol use. Mindfulness (N Y). 2012;3(4):308-17.

34. Henrique IFS, De Micheli D, Lacerda RB, Lacerda LA, Formigoni MLOS. Validação da versão brasileira do teste de triagem do envolvimento com álcool, cigarro e outras substâncias (ASSIST). Rev Assoc Med Bras. 2004;50(2):199-206.

35. Barros VV, Kozasa EH, Souza ICW, Ronzani TM. Validity evidence of the Brazilian version of the Mindful Attention Awareness Scale (MAAS). Psicol Reflex Crít. 2015;28(1):87-95.

36. Drobes DJ. Cue reactivity in alcohol and tobacco dependence. Alcohol Clin Exp Res. 2002;26(12):1928-9. 
37. Correia GAR, Gondim APS. Utilização de benzodiazepínicos e estratégias farmacêuticas em saúde mental. Saúde em Debate. 2014;38(101):393-8.

38. Davies J, Rae TC, Montagu L. Long-term benzodiazepine and Z-drugs use in England: a survey of general practice. Br J Gen Pract. 2017;67(662):e609-13.

39. Ives-Deliperi VL, Solms M, Meintjes EM. The neural substrates of mindfulness: An fMRI investigation. Soc Neurosci. 2011;6(3):231-42.

40. Bowen S, Marlatt A. Surfing the Urge: Brief Mindfulness-Based Intervention for College Student Smokers. Psychol Addict Behav. 2009;23(4):666-71.
41. Wilson JD, Vo H, Matson P, Adger H, BarnettG, Fishman M. Traitmindfulness and progression to injection use in youth with opioid addiction. Subst Use Misuse. 2017;52(11):1486-93.

42. Field M, Cox W. Attentional bias in addictive behaviors: A review of its development, causes, and consequences. Drug Alcohol Depend. 2008;97(1-2):1-20

43. Curado D, Barros V, Opaleye E, Bowen S, Hachul H, Noto AR. The role of mindfulness in the insomnia severity of female chronic hypnotic users. Int J Behav Med. 2018;25(5):526-31. 